\title{
PARTIAL FRACTION DECOMPOSITIONS AND EXPANSIONS OF ZERO ${ }^{1}$ )
}

\author{
BY
}

\section{JOSEPH LEHNER}

1. In 1936 Rademacher found an expression for the partition function $p(n)$ in the form of a convergent series:

$$
p(n)=\sum_{h, k} \underset{h k}{-n} \epsilon_{h k}^{-n} g_{k}(n), \quad k=1,2, \cdots ; h \bmod k,(h, k)=1
$$

where

$$
\alpha_{h k}=k^{1 / 2} \omega_{h k} / \pi 2^{1 / 2},
$$

$\omega_{h k}$ being a complicated root of unity, $\epsilon_{h k}=\exp (2 \pi i h / k)$, and

$$
g_{k}(z)=\frac{d}{d z}\left\{\frac{\sinh C \lambda(z) / k}{\lambda(z)}\right\}, \quad \lambda(z)=(z-1 / 24)^{1 / 2}
$$

is an entire function of $z$ of order $1 / 2$.

The generating function of $p(n)$ is

$$
f(x)=\prod_{1}^{\infty}\left(1-x^{m}\right)^{-1}=1+\sum_{1}^{\infty} p(n) x^{n}, \quad|x|<1 .
$$

Rademacher inserted the series for $p(n)$ into (1.2) and obtained in this way a new representation of $f(x)$ :

$$
f(x)=1+\sum_{h, k} \alpha_{h k} \Phi_{k}\left(x / \epsilon_{h k}\right) .
$$

Here

$$
\Phi_{k}(x)=\sum_{n=0}^{\infty} g_{k}(n) x^{n}
$$

is, by virtue of the properties $\left({ }^{2}\right)$ of $g_{k}(z)$, an analytic function of $x$ which is regular everywhere (incl. $\infty$ ) except at $x=1$. Thus the natural boundary $(|x|=1)$ of $f(x)$ is here represented as made up of contributions from "generalized partial fractions," $\Phi_{k}\left(x / \epsilon_{h k}\right)$, one for each root of unity $\epsilon_{h k}$.

Received by the editors September 10, 1956.

(1) Work performed under the auspices of the Atomic Energy Commission. This paper was read by title at the Number Theory Conference (Pasadena, June, 1955).

(2) This holds under the more general condition that $g_{k}(z)$ is at most of the zero type of order 1 , by Wigert's theorem. See beginning of $\$ 2$. 
Rademacher also studied the series in (1.3) when $|x|>1$. He found that it converged uniformly and defined a function

$$
f^{*}(x)=1+\sum_{h, k} \alpha_{h k} \Phi_{k}\left(x / \epsilon_{h k}\right)=-\sum_{1}^{\infty} p(-n) x^{-n}, \quad|x|>1
$$

where $p(-n)$ is the expression obtained by formally replacing $n$ by $-n$ in the series for $p(n)$. Rademacher conjectured $[1$, p. 84$]$ and later proved $\left({ }^{3}\right)$ the surprising fact that $p(-n)=0, n=1,2, \cdots$; in other words that

$$
f^{*}(x) \equiv 0 \text {. }
$$

Equation (1.6) is called an "expansion of zero." Equations of this type involving modular forms $(f(x)$ is essentially a modular form) were known to Poincaré $\left({ }^{4}\right)$.

The object of this paper is to generalize the situation described above. For $\left\{g_{k}(z)\right\}$ we shall take entire functions of class $(1,0)$, i.e., functions belonging to at most the minimum type of order 1 . We assume that

$$
\limsup _{n}\left(\sum_{k=0}^{\infty}\left|g_{k}(n)\right|\right)^{1 /|n|} \leqq 1,
$$

where the lim sup is taken over all integral $n$. Let $\left\{\epsilon_{k}\right\}, k=0,1,2, \cdots$ be a set of arbitrary, distinct complex numbers of absolute value 1 , and $\left\{\alpha_{k}\right\}$, $k=0,1,2, \cdots$, a set of bounded numbers. Then, defining $\Phi_{k}(x)$ by (1.4), we prove that $\Phi_{k}(x)$ can be continued to the exterior of the unit circle and that the series

$$
\Sigma=\sum_{k=0}^{\infty} \alpha_{k} \Phi_{k}\left(x / \epsilon_{k}\right)
$$

converges uniformly both inside and outside the unit circle. The Taylor coefficients of the two analytic functions defined by $\Sigma$ are given by the formula

$$
a_{n}=\sum_{k=0}^{\infty} \alpha_{k} \epsilon_{k}^{-n} \cdot g_{k}(n), \quad n=0, \pm 1, \pm 2, \cdots .
$$

This is Theorem 1(\$2). It includes Rademacher's example, for we have only to replace $\alpha_{h k}$ by $\omega_{h k} / \pi 2^{1 / 2}, g_{k}(n)$ by $k^{1 / 2} g_{k}(n)$, and $(h, k)$ by an index which runs over all the rational numbers in $(0,1)$. Condition $(1.7)$ is seen to be satisfied for $\left.{ }^{5}\right)\left\{k^{1 / 2} \phi(k) g_{k}(n)\right\}$.

Theorem 2 is a converse theorem. We assume there is an expansion of the type (1.8) converging inside and outside the unit circle. We then show the

(3) Unpublished. The only published proof is in Petersson [2].

(4) A discussion of other types of expansions of zero can be found in [6].

(5) The factor $\phi(k)$ appears because each $g_{k}(n)$ occurs $\phi(k)$ times as $h \operatorname{runs} \bmod k,(h, k)=1$. 
existence of functions $\left\{g_{k}(z)\right\}$ connected with $\Phi_{k}(x)$ by (1.4) and satisfying (1.9) and (1.7).

In $\S 3$ we consider the possibility of continuing the series (1.8) analytically across the unit circle. If the set $\left\{\boldsymbol{\epsilon}_{k}\right\}$ is not dense in the unit circle, there is an arc on which the function defined by the series will be regular, provided a certain condition, (3.3), (possibly stronger than (1.7)) is satisfied by $\left\{g_{k}(z)\right\}$. Then the analytic functions defined by the series inside and outside the unit circle are continuations of each other. The proof is made by finding a representation of $\Phi_{k}(x)$ which is perhaps of some interest in itself.

$\$ 4$ is devoted to the inverse problem: given a suitably restricted function $G(x)$ regular in the unit circle, is there a series (1.8) which represents $G(x)$ ? This amounts to solving the infinite system (1.9) for $\left\{\alpha_{k}\right\}$ when $\left\{a_{n}\right\}$ is given $(n \geqq 0)$, which can be done by treating this system as a linear mapping between Banach spaces. We assume $G(x)$ has bounded Taylor coefficients, and find in Theorem 4 a sufficient condition on $\left\{g_{k}(z)\right\}$.

Finally, in $\$ 5$, Theorem 4 is used to develop series of type (1.8) which represent zero identically in the interior of the unit circle.

2. The developments of this section depend on

WigERT'S THEOREM. If $g(z)$ is a function of class $(1,0)$, then the function defined by the series

$$
f(x)=c+\sum_{n=1}^{\infty} g(x) x^{n}, \quad|x|<1
$$

and its analytic continuation is regular in the whole plane (incl. $\infty$ ) except at $x=1$. Conversely, if $f(x)$ is a function with the above regularity properties then there is a function $g(z)$ of class $(1,0)$ such that $(2.1)$ holds in $|x|<1$. If $g(z)$ is a polynomial, $f(x)$ is a rational function of $1 /(1-x)$, and conversely.

For a proof, see [3, pp. 297-300]. By a function $g(z)$ of class $(1,0)$, we mean an entire function belonging to at most the zero type of order 1, i.e., $g(z)$ satisfies

$$
\max _{\theta}\left|g\left(r e^{i \theta}\right)\right|<e^{\epsilon r}, \quad r>r_{0}(\epsilon)
$$

for every $\epsilon>0$.

We need the following slight extension of Wigert's theroem.

LeMma 1. If $g(z)$ is a function of class $(1,0)$, and

$$
f(x)=\sum_{n=0}^{\infty} g(n) x^{n}
$$

then $f(\infty)=0$. If $f(x)$ is regular except at $x=1$, there is a $g(z)$ of class $(1,0)$ such that (2.2) holds. 
The proof of this lemma will be made in $\S 3$.

We also require a few results on sequence spaces. Let $B$ be the Banach space of bounded sequences $x=\left(x_{0}, x_{1}, x_{2}, \cdots\right)$ with

$$
\|x\|=\sup _{k}\left|x_{k}\right| \text {. }
$$

Let $B^{\prime}$ be the Banach space of bounded doubly-infinite sequences $y$ $=\left(\cdots, y_{-2}, y_{-1}, y_{0}, y_{1}, y_{2}, \cdots\right)$ with the same norm. We consider a linear transformation $T$ from $B$ into $B^{\prime}$ defined by an infinite matrix $\left(t_{n k}\right),-\infty<n$ $<\infty, k \geqq 0$ :

$$
T: y_{n}=\sum_{k=0}^{\infty} t_{n k} x_{k}
$$

Denote the norm of $T$ by $|T|$; as usual we have

$$
|T|=\sup _{\|x\|=1}\|T x\| \text {. }
$$

Concerning the Transformation $T$, we wish to prove two lemmas.

Lemma 2a. A necessary and sufficient condition that $T$ in (2.3), should define a linear transformation from $B$ into $B^{\prime}$ is that

$$
\sup _{n} \sum_{k=0}^{\infty}\left|t_{n k}\right|<\infty \text {. }
$$

If this condition is satisfied, $T$ is a bounded transformation and $|T|$ is equal to the constant (2.4).

The lemma implies, in particular, that every linear transformation on $B$ to $B^{\prime}$ of the form (2.3) is bounded. To prove it, let $T$ be from $B$ to $B^{\prime}$. Define the linear functionals

and the vectors, of norm one,

$$
\phi_{n}(x)=\sum_{k=0}^{\infty} t_{n k} x_{k}
$$
all $n$,

$$
x^{(n)}=\left\{\begin{array}{cl}
\left\{\left|t_{n k}\right| / t_{n k}\right\}, & t_{n k} \neq 0, \\
1, & t_{n k}=0 .
\end{array}\right.
$$

By hypothesis $\left\{\phi_{n}(x)\right\}$ is bounded when $x=\left\{x_{k}\right\}$ is bounded; in particular $\phi_{n}\left(x^{(n)}\right)$ is finite. I.e.,

$$
\phi_{n}\left(x^{(n)}\right)=\sum_{k=0}^{\infty}\left|t_{n k}\right|=A_{n}<\infty .
$$

This implies that, for each $n, \phi_{n}(x)$ is a bounded linear functional, for

(e) The expression "all $n$ " shall mean that $n$ assumes all integral values. 


$$
\left|\phi_{n}(x)\right| \leqq \sum_{k=0}^{\infty}\left|t_{n k}\right|\left|x_{k}\right| \leqq A_{n}\|x\| .
$$

Also, $\sup _{n}\left|\phi_{n}(x)\right|<\infty$ for all $x \in B$, since $\left\{\phi_{n}(x)\right\} \in B^{\prime}$. It follows from Banach's theorem on functionals $[4$, p. 25] that there is an $A$ such that $\left|\phi_{n}(x)\right| \leqq A$ for all $n$ and all $x \in B,\|x\| \leqq 1$. Hence,

$$
\phi_{n}\left(x^{(n)}\right)=\sum_{k}\left|t_{n k}\right| \leqq A,
$$

as required.

Now

$$
\begin{aligned}
\sup _{n} \sum_{k=0}^{\infty}\left|t_{n k}\right| & \geqq \sup _{\|x\|=1} \sup _{n}\left|\sum_{k} t_{n k} x_{k}\right|=\sup _{\|x\|=1}\|T x\|=|T| \\
& \geqq \sup _{l}\left\|T x^{(l)}\right\|=\sup _{l} \sup _{n}\left|\sum_{k} t_{n k} x_{k}^{(l)}\right| \\
& \geqq \sup _{n}\left|\sum_{k} t_{n k} x_{k}^{(n)}\right|=\sup _{n} \sum_{k}\left|t_{n k}\right| .
\end{aligned}
$$

If (2.4) is fulfilled, $|T|<\infty$, and the proof is complete.

Lemma 2b. If $T$ is given by (2.3), and

$$
\sup _{n} \sum_{k=0}^{\infty}\left|t_{n k}-\delta_{n k}\right|<1,
$$

$T^{-1}$ exists as a bounded linear transformation from $B^{\prime}$ into $B$.

By the preceding lemma, $T$ is a bounded transformation on $B$ to $B^{\prime}$, for

$$
|T|=\sup _{n} \sum_{k}\left|t_{n k}\right| \leqq 1+\sup _{n} \sum_{k}\left|t_{n k}-\delta_{n k}\right|<2 .
$$

Also $|T-1|<1$. The proof is completed by quoting a known result:

If $T$ is a bounded linear operator from a Banach space $X$ into a Banach space $Y$ such that $|T-1|<1$, then $T^{-1}$ exists and is a bounded operator from $Y$ into $X$. See $[5$, p. 52] where the proof is given for $X=Y=$ Hilbert space, but the same proof applies to the present case.

We come now to the proofs of the theorems mentioned in $\S 1 . R_{1}, R_{2}$ shall denote the regions $|x|<1,|x|>1$, respectively, and $Q$ the unit circle $|x|=1$. By $\Sigma$ we shall mean the series (1.8).

THeOREM 1. Let $g_{k}(z), k=1,2,3, \cdots$, be functions of class $(1,0)$. Let $\left\{\epsilon_{k}\right\}$ be distinct complex constants of modulus one. Let the series $\sum_{k=0}^{\infty}\left|g_{k}(n)\right|$ converge for all $n$, and

$$
\limsup _{n}\left(\sum_{k=0}^{\infty}\left|g_{k}(n)\right|\right)^{1 /|n|} \leqq 1 .
$$


Then if $\left\{\alpha_{k}\right\}$ is any bounded sequence, the series $\Sigma$ converges uniformly on every set $S$ at a positive distance from $Q$, and defines two regular functions

$$
G_{1}(x)=\sum_{0}^{\infty} a_{n} x^{n}, x \in R_{1} ; \quad G_{2}(x)=-\sum_{1}^{\infty} a_{-n} x^{-n}, x \in R_{2}
$$

where, for all $n$,

$$
a_{n}=\sum_{k=0}^{\infty} \alpha_{k} \epsilon_{k}^{-n} g_{k}(n)
$$

$\Phi_{k}(x)$ is defined by (1.4).

Proof. By Wigert's theorem, the functions $\Phi_{k}(x)$ are defined in the whole plane and are everywhere regular (incl. $\infty$ ) except at $x=1$. The hypothesis (2.7) implies

$$
\sum_{k=0}^{\infty}\left|g_{k}(n)\right| \leqq A_{\epsilon} \epsilon^{\epsilon|n|}
$$

all $n$;

hence,

$$
\left|g_{k}(n)\right| \leqq A_{\epsilon} e^{e|n|}, \quad \text { all } n, k \geqq 0 .
$$

We use this result for non-negative $n$ to provide a uniform estimate of $\Phi_{k}(x)$ :

$$
\left|\Phi_{k}(x)\right| \leqq \sum_{n=0}^{\infty}\left|g_{k}(n)\right||x|^{n} \leqq A_{\epsilon}\left(1-\left|x e^{\epsilon}\right|\right)^{-1}, \quad\left|x e^{\epsilon}\right|<1 .
$$

Since $\left\{\alpha_{k}\right\}$ is bounded, this proves the uniform convergence of the series $\Sigma$ on every subset of $R_{1}$ at a positive distance from $Q$.

The convergence of $\Sigma$ in $R_{2}$ is next discussed. We have from (1.4)

$$
g_{k}(n)=\frac{1}{2 \pi i} \int_{|\zeta|=\eta} \Phi_{k}(\zeta) \zeta^{(n+1)} d \zeta, \quad n \geqq 1 .
$$

Define $\left({ }^{7}\right)$

$$
I_{k}(t)=-\frac{1}{2 \pi i} \int_{|\zeta-1|=\eta} \Phi_{k}(\zeta) \zeta^{(t+1)} d \zeta, \quad \eta<1, \zeta^{t}=e^{t \log \zeta}
$$

where $\log 1=0$. Since $\log \zeta$ is uniform on the path of integration, we find that $I_{k}(t)$ is an entire function of $t$, and indeed is of class $(1,0)$. For

$$
\left|I_{k}(t)\right| \leqq \max _{|\zeta-1|=\eta}\left|\Phi_{k}(\zeta)\right| \cdot \eta e^{2 \eta|t+1|}=A e^{2 \eta|t|}
$$

for every $0<\eta<1 / 2$. Now

(7) Cf. [3, p. 298]. 


$$
I_{k}(n)=\frac{1}{2 \pi i} \int_{|\zeta|=\eta} \Phi_{k}(\zeta) \zeta^{-(n+1)} d \zeta, \quad n=1,2,3, \cdots,
$$

for when $R$ is large enough

$$
\left|\frac{1}{2 \pi i} \int_{|\zeta|=R} \Phi_{k}(\zeta) \zeta^{-(n+1)} d \zeta\right| \leqq 2\left|\Phi_{k}(\infty)\right| \cdot R^{-n} \rightarrow 0, \quad R \rightarrow \infty .
$$

Thus, by comparison of (2.12) and (2.13), we see that $I_{k}(t)=g_{k}(t)$ on the positive integers, and hence, by a known theorem, everywhere $\left(^{8}\right)$. In particular for large $R$ and $n=1,2,3, \cdots$,

$$
g_{k}(-n)=-\frac{1}{2 \pi i} \int_{|\zeta-1|=\eta} \Phi_{k}(\zeta) \zeta^{n-1} d \zeta=-\frac{1}{2 \pi i} \int_{|\zeta|=R} \Phi_{k}(\zeta) \zeta^{n-1} d \zeta,
$$

which implies

$$
\Phi_{k}(x)=-\sum_{1}^{\infty} g_{k}(n) x^{-n}, \quad x \in R_{2}
$$

since, by Lemma $1, \Phi_{k}(\infty)=0$.

The last equation furnishes the continuation of $\Phi_{k}(x)$ to the outside of the unit circle. Again from (2.10) we obtain the estimate

$$
\left|\Phi_{k}(x)\right| \leqq \frac{A_{\epsilon}}{1-\left|x^{-1} e^{\epsilon}\right|}, \quad\left|x e^{-\epsilon}\right|>1,
$$

from which the uniform convergence of $\Sigma$ on the required subsets of $R_{2}$ follows.

Let $x \in R_{1}$. We have

$$
\Sigma=\sum_{k=0}^{\infty} \alpha_{k} \sum_{n=0}^{\infty} g_{k}(n) \epsilon_{k}^{-n} x^{n}=\sum_{n=0}^{\infty} x^{n} \sum_{k=0}^{\infty} \alpha_{k} \epsilon_{k}^{-n} g_{k}(n)=\sum_{n=0}^{\infty} a_{n} x^{n}
$$

The interchange of order is justified by (2.10):

$$
\sum_{n=0}^{\infty}|x|^{n} \sum_{k=0}^{\infty}\left|\alpha_{k}\right|^{n}\left|g_{k}(n)\right| \leqq \sum_{n=0}^{\infty}|x|^{n} \cdot M A_{\epsilon} e^{\epsilon|n|}<\infty
$$

where $\left|\alpha_{k}\right|<M, k=0,1,2, \cdots$. This proves (2.9) when $n \geqq 0$; when $n<0$ we proceed in the same way using (2.14).

Next, we prove a converse theorem.

THEOREM 2. Suppose we are given that $\Sigma$ converges uniformly on every set $S$ at a positive distance from $Q$, where $\left\{\boldsymbol{\epsilon}_{k}\right\}$ is a fixed sequence of distinct com-

${ }^{(8)}$ This result actually follows from Wigert's theorem. Suppose $\Delta(z)$ is of class $(1,0)$ and $\Delta(n)=0, n=1,2,3, \cdots$. Then $\Psi(x)=\sum_{0}^{\infty} \Delta(n) x^{n}=\Delta(0)$. I.e., $\Psi$ is a rational function of $(1-x)^{-1}$ and so, by the converse part of Wigert's theorem, $\Delta(z)$ is a polynomial. Since $\Delta$ vanishes on the positive integers, it vanishes identically. 
plex numbers of modulus one, $\left\{\Phi_{k}(x)\right\}$ is a fixed sequence of functions regular everywhere except at $x=1, \Phi_{k}(\infty)=0$, and $\left\{\alpha_{k}\right\}$ is any bounded sequence. Then there exist functions $\left\{g_{k}(z)\right\}$ of class $(1,0)$ and constants $\left\{a_{n}\right\}$ such that $(1.4)$, (2.7)-(2.9), and (2.14) hold.

Proof. By hypothesis $\Sigma$ converges uniformly and, therefore, defines two regular functions:

$$
\Sigma=G_{1}(x), x \in R_{1} ; \quad \Sigma=G_{2}(x), x \in R_{2} .
$$

In particular,

$$
\lim _{x \rightarrow \infty} G_{2}(x)=\lim _{x \rightarrow \infty} \sum_{0}^{\infty} \alpha_{k} \Phi_{k}\left(x / \epsilon_{k}\right)=0,
$$

since $\Phi_{k}(\infty)=0$. Put

$$
G_{1}(x)=\sum_{0}^{\infty} a_{n} x^{n}, x \in R_{1} ; \quad G_{2}(x)=\sum_{n=1}^{\infty} a_{-n} x^{-n}, x \in R_{2} .
$$

this being a definition of $\left\{a_{n}\right\}$. By repeated differentiation of (2.16) we find

$$
G_{1}^{(n)}(0)=n ! a_{n}=\sum_{0}^{\infty} \alpha_{k} \epsilon_{k}^{-n} \Phi_{k}^{(n)}(0), \quad n=0,1,2, \cdots .
$$

Now by Lemma 1 and the argument following (2.12), there is associated with each $\Phi_{k}(x)$ a function $g_{k}(z)$ of class $(1,0)$ such that (1.4) and (2.14) hold. Differentiating (1.4) gives

$$
\Phi_{k}^{(n)}(0)=n ! g_{k}(n), \quad \quad n=0,1,2, \cdots
$$

and substituting this into the preceding equation we get the expression (2.9) for $a_{n}$ when $n \geqq 0$. The discussion for $n<0$ is similar using (2.14) instead of (1.4).

It follows from the convergence of $G_{1}$ and $G_{2}$ in their respective regions that

$$
\limsup _{n}\left|a_{n}\right|^{1 /|n|} \leqq 1 .
$$

Multiply (2.9) by $e^{-\epsilon|n|}, \epsilon>0$ :

$$
\sum_{0}^{\infty} \alpha_{k}\left\{e^{-\epsilon|n|-n} \epsilon_{k}^{-n} g_{k}(n)\right\}=a_{n} e^{-\epsilon|n|},
$$

all $n$.

By (2.18) the right member is bounded in $n$, and so the matrix $\left(\epsilon_{k}^{-n} e^{-\epsilon|n|} g_{k}(n)\right)$ defines a linear transformation from $B$ into $B^{\prime}$. Lemma 2 a now shows that

$$
\sup _{n} \sum_{0}^{\infty} e^{-\epsilon|n|}\left|\epsilon_{k}^{-n} g_{k}(n)\right|=\sup _{n} e^{-\epsilon|n|} \sum_{0}^{\infty}\left|g_{k}(n)\right|=A_{\epsilon}<\infty .
$$


Since this holds for each $\epsilon>0$, we have (2.7)

3. Define

$$
M=\text { closure of }\left\{\epsilon_{k}\right\}, \quad k=0,1,2, \cdots .
$$

If $M=Q$ there is nothing further to be said; $\Sigma$ converges wherever it can be expected to. However, if $M \neq Q$ there is an arc on $Q$ which is free of points of $M$. Under certain conditions, the series converges on this arc also.

Let

$$
g_{k}(z)=\sum_{j=0}^{\infty} c_{k_{j}} z^{j}, \quad k=0,1,2, \cdots
$$

We shall impose the condition

$$
\sum_{k=0}^{\infty}\left|c_{k_{j}}\right| \leqq A_{\epsilon}(\epsilon /(j+1))^{j}, \quad j=0,1,2, \cdots .
$$

THEOREM 3. If the hypotheses of Theorem 1 are fulfilled, and if, in addition, (3.3) is satisfied, then $\Sigma$ converges uniformly on every subset of $Q-M$ at a positive distance from $M$, and the functions $G_{1}, G_{2}$ of (2.7) are analytic continuations of each other.

REMARK. If $c_{k_{j}} \geqq 0$, or if $c_{k_{j}}=\alpha_{k} \beta_{j}$, it is easy to see that (3.3) follows from the hypotheses in Theorem 1 . Whether this is true in general, the author is unable to decide.

Proof. By hypothesis, both (3.3) and (3.4) are satisfied. Let $x \in R_{1}$, then

$$
\Phi_{k}(x)=\sum_{n=0}^{\infty} g_{k}(n) x^{n}=\sum_{n=0}^{\infty} x^{n} \sum_{j=0}^{\infty} c_{k_{j}} n^{i}=\sum_{j=0}^{\infty} c_{k_{j}} \sum_{n=0}^{\infty} n^{i} x^{n},
$$

the inversion being justified by (3.3). Define

$$
\phi_{j}(x)=\sum_{n=0}^{\infty} n^{i} x^{n}, \quad j=0,1,2, \cdots, x \in R_{1}
$$

so that the above equation may be written

$$
\Phi_{k}(x)=\sum_{j=0}^{\infty} c_{k j} \phi_{j}(x), \quad k=0,1,2, \cdots, x \in R_{1} .
$$

We now prove two lemmas.

Lemma 3. For $w \neq 1$ we have

$$
\phi_{j}(w)=\sum_{l=1}^{j+1} \frac{A_{l_{j}}}{(1-w)^{l}}, \quad j \geqq 0
$$




$$
A_{l_{j}}=(-1)^{j+1} \sum_{\nu=1}^{l}(-1)^{\nu} C_{l-1, \nu-1} \nu^{j}, \quad j \geqq 0 .
$$

As usual we put $C_{m, n}=0, n>m, C_{m, 0}=1, m \geqq 0$.

Proof. Define $B_{l_{j}}, l \geqq 0 ; j \geqq 0$ by

$$
\phi_{j}(w)=\sum_{l=1}^{\infty} \frac{B_{l_{j}}}{(1-w)^{l}}, \quad B_{0 j}=0 .
$$

From the definition (3.5) we have the recursion

$$
\phi_{j+1}(w)=w \phi_{j}^{\prime}(w),
$$

This makes it clear that $\phi_{j}(w)$ is a finite sum of terms of the form (3.7). Hence in (3.9), we have $B_{l_{j}}=0, l>j+1$, the series converges, and we can differentiate term by term in (3.10). This gives

$$
B_{l, j+1}=-l B_{l_{j}}+(l-1) B_{l-1, j}, \quad l \geqq 1, j \geqq 0
$$

where we have used $B_{0_{j}}=0$. Our first object is to show that the $B_{l_{j}}$ have the values $A_{l_{j}}$.

We first prove, by induction on $j$, that $\left\{A_{l_{j}}\right\}$ satisfies the recursion (3.11). Let $j=0 . A_{l_{0}}=\delta_{1 l}$. We must verify that

$$
\sum_{\nu=1}^{l}(-1) \nu C_{l-1, \nu-1} \nu=-l \delta_{1 l}+(l-1) \delta_{1, l-1}, \quad l \geqq 1 .
$$

For $l=1,2$ we easily calculate that both members have the values $-1,+1$, respectively. When $l=2$, the right member obviously vanishes, while the left member is

$$
\sum_{\nu=1}^{l}(-1)^{\nu} C_{l-1, \nu-1}+\sum_{\nu=2}^{l}(-1)^{\nu} C_{l-1, \nu-1}(\nu-1)=0+(l-1) \sum_{\nu=2}^{l}(-1)^{\nu} C_{l-2, \nu-2}=0 .
$$

Now suppose that $\left\{A_{l_{j}}\right\}$ satisfies (3.11) for $j \leqq k-1$, where we may take $k \geqq 1$. Putting $j=k, l \geqq 2$ we have

$$
\begin{aligned}
-l A_{l k} & =(-1)^{k} \sum_{\nu=1}^{l}(-1)^{\nu} l C_{l-1, \nu-1} \nu^{k}=(-1)^{k} \sum_{\nu=1}^{l}(-1)^{\nu} C_{l, \nu \nu^{b+1}} \\
& =(-1)^{k} \sum_{\nu=1}^{l}(-1)^{\nu}\left\{C_{l-1, \nu-1}+C_{l-1, \nu-1}\right\} \nu^{k+1} \\
& =A_{l, k+1}+(-1)^{k}(l-1) \sum_{\nu=1}^{l-1}(-1)^{\nu} C_{l-1, \nu-1} \nu^{k}=A_{l, k+1}-(l-1) A_{l-1, k} .
\end{aligned}
$$

When $l=1$, (3.11) reduces to the easily checked formula $A_{1, k+1}=-A_{1, k}$. Hence $\left\{A_{l_{j}}\right\}$ satisfies $(3.10)$ for all $l \geqq 1, j \geqq 0$. 
Next, $A_{l_{0}}=B_{l_{0}}, l \geqq 1$, i.e., $B_{l_{0}}=\delta_{1 l}$. This is a trivial consequence of (3.5) and (3.9).

This completes the proof that the $B_{l_{j}}$, defined by (3.9), have the values (3.8) when $1 \leqq l \leqq j+1$. Since, as already noted, $B_{l_{j}}=0$ for $l>j+1$, it is evident that $B_{l_{j}} \equiv A_{l_{j}}, l \geqq 1, j \geqq 0$, and the lemma is established.

Lemma 4 . For $j \geqq 1,|1-w| \geqq \epsilon>0(\epsilon<2)$, we have

$$
\left|\phi_{j-1}(w)\right| \leqq(2 j / \epsilon)^{j} \text {. }
$$

From the preceding lemma we have

$$
\left|A_{l_{j}}\right| \leqq \sum_{1}^{l} C_{l-1, \nu-1 \nu^{j}} \leqq 2^{l-1} l^{j}
$$

hence, by (3.7),

$$
\left|\phi_{j-1}(w)\right| \leqq \sum_{l=1}^{j} \frac{2^{l-1} l^{j-1}}{|1-w|^{l}} \leqq \sum_{1}^{j}\left(\frac{2}{\epsilon}\right)^{l} l^{j-1} \leqq\left(\frac{2 j}{\epsilon}\right)^{\prime} .
$$

Lemma 4 shows that in (3.6) the series converges for all $w \neq 1$. For (3.3) implies that

$$
\left|c_{k_{j}}\right| \leqq A_{\epsilon}\left(\frac{\epsilon}{j}\right)^{\prime}, \quad j=1,2,3, \cdots, k=0,1,2, \cdots,
$$

so

$$
\begin{aligned}
\sum_{j=1}^{\infty}\left|c_{k_{j}}\right|\left|\phi_{j}(x)\right| & \leqq A_{\epsilon / 8} \sum_{j}\left(\frac{2(j+1)}{\epsilon}\right)^{j+1}\left(\frac{\epsilon}{8 j}\right)^{\prime} & \\
& \leqq \frac{4}{\epsilon} A_{\epsilon / 8} \sum_{j} j \cdot 2^{-j}, & |1-w| \geqq \epsilon
\end{aligned}
$$

and the right member is finite for each $\epsilon>0$. Hence, (3.6) is valid for all $x \neq 1$.

We can now prove Theorem 3. Let $S$ be a subset of $Q-M$, and $d(0<d<2)$ be the distance from $S$ to $M$. If $x \in S$, we have $\left|x / \epsilon_{k}-1\right| \geqq d, k=0,1,2, \cdots$. Hence, using (3.6) and (3.3) we get

$$
\begin{aligned}
\sum_{k=0}^{\infty}\left|\Phi_{k}\left(x / \epsilon_{k}\right)\right| & \leqq \sum_{k} \sum_{j=0}^{\infty}\left|c_{k_{j}}\right|\left|\phi_{j}\left(x / \epsilon_{k}\right)\right| \\
& \leqq \sum_{j}\left(\frac{2(j+1)}{d}\right)^{j+1} \sum_{k}\left|c_{k_{j}}\right| \leqq \frac{2}{d} A_{\epsilon}+A_{\epsilon} \sum_{1}^{\infty}\left(\frac{4 \epsilon}{d}\right)^{j+1}<\infty
\end{aligned}
$$

if we choose $4 \epsilon / d<1$. Since $\left\{\alpha_{k}\right\}$ is bounded, this proves that $\Sigma$ converges uniformly on $S$. The last statement of the theorem is obvious.

It is convenient at this point to prove Lemma 1. In analogy with (3.6) we are permitted to write 


$$
f(x)=\sum_{j=0}^{\infty} c_{j} \phi_{j}(x)
$$

In view of (3.7) we have $\lim _{x \rightarrow \infty} \phi_{j}(x)=0, j \geqq 0$. Because of the uniform convergence of the series in (3.14) we now have $\lim _{x \rightarrow \infty} f(x)=0$. Conversely, if $f(x)$ is regular except at $x=1$, we have, by Wigert's theorem,

$$
\begin{aligned}
f(x) & =c+\sum_{1}^{\infty} g(n) x^{n}=c-g(0)+\sum_{0}^{\infty} g(n) x^{n}, & & x \in R_{1} \\
& =c-g(0)+\sum_{j=0}^{\infty} c_{j} \phi_{j}(x), & & x \neq 1 .
\end{aligned}
$$

Letting $x \rightarrow \infty$, we see by the argument just made that $c=g(0)$.

4. This section will be devoted to a proof of

THEOREM 4. Let $G(x)$ be an analytic function, regular in $R$, having bounded Taylor coefficients. Let $\left\{\epsilon_{k}\right\}, k=0,1,2, \cdots$, be arbitrary complex numbers of modulus one. If

$$
\sup _{n \geqq 0} \sum_{k=0}^{\infty}\left|\epsilon_{k}^{-n} g_{k}(n)-\delta_{k n}\right|=\kappa<1,
$$

there exists a bounded sequence $\left\{\alpha_{k}\right\}$ such that

$$
\Sigma=G(x), \quad x \in R_{1} .
$$

The conditions of the theorem are fulfilled, for example, by the set

$$
\begin{array}{lll}
g_{k}(z)=\frac{1}{2} C_{z, k}\left(\frac{\sin (8 k(z-k))^{1 / 2}}{(8 k(z-k))^{1 / 2}}\right)^{2 k}, & k>0, \\
g_{0}(z)=\frac{1}{4} ; \quad \epsilon_{k}=\exp 2 \pi i / k, k>0 ; \quad \epsilon_{0}=1 &
\end{array}
$$

in which case an upper bound for $\kappa$ is $9 / 10$.

To prove the theorem, consider the system

$$
\sum_{k=0}^{\infty} \alpha_{k} \epsilon_{k}^{-n} g_{k}(n)=a_{n}, \quad n \geqq 0
$$

where $G(x)=\sum_{n=0}^{\infty} a_{n} x^{n}$. Denoting the matrix of the system by $T=\left(t_{n k}\right)$, we see that the conditions of Lemma $2 \mathrm{~b}\left({ }^{9}\right)$ are satisfied in view of (4.1), and therefore $T^{-1}$ exists. Since $\left\{a^{n}\right\}$ is bounded by hypothesis, the system (4.2) is solved by $\alpha=T^{-1} a$, where $\alpha=\left(\alpha_{0}, \alpha_{1}, \cdots\right), a=\left(a_{0}, a_{1}, \cdots\right)$.

Multiply (4.2) by $x^{n}$ and sum. We get for $x \in R_{1}$, itself.

( ${ }^{9}$ We apply the obvious modification of this lemma which refers to mappings from $B$ into 


$$
\begin{aligned}
\sum_{n=0}^{\infty} a_{n} x^{n} & =G(x)=\sum_{n=0}^{\infty} x^{n} \sum_{k=0}^{\infty} \alpha_{k} \epsilon_{k}^{-n} g_{k}(n) \\
& =\sum_{k=0}^{\infty} \alpha_{k} \sum_{n=0}^{\infty} g_{k}(n)\left(x / \epsilon_{k}\right)^{n}=\sum_{k=0}^{\infty} \alpha_{k} \Phi_{k}\left(x / \epsilon_{k}\right)=\Sigma .
\end{aligned}
$$

The interchange of summation orders must be justified:

$$
\sum_{k=0}^{\infty}\left|g_{k}(n)\right|=\sum_{0}^{\infty}\left|\epsilon_{k}^{-n} g_{k}(n)\right| \leqq \sum_{0}^{\infty}\left|\epsilon_{k}^{-n} g_{k}(n)-\delta_{k n}\right|+1 \leqq \kappa+1
$$

for $n \geqq 0$; hence, if $\left|\alpha_{k}\right| \leqq A$, we have

$$
\sum_{k, n}\left|\alpha_{k}\right|\left|g_{k}(n)\right|\left|x / \epsilon_{k}\right|^{n} \leqq A \sum_{n}|x|^{n} \sum_{k}\left|g_{k}(n)\right| \leqq 2 A \sum_{n}|x|^{n}<\infty .
$$

This completes the proof.

Incidentally, a bound on $\left\{\alpha_{k}\right\}$ is easily obtained:

$$
|\alpha|=\sup _{k}\left|\alpha_{k}\right| \leqq\left|T^{-1}\right|\|a\| \leqq \sum_{\nu=0}^{\infty}|1-T| \nu\|a\|=\frac{\|a\|}{1-\kappa},
$$

since (4.1) is equivalent to $|1-T|=\kappa$.

5. We can use the developments of the preceding sections to obtain a class of expansions of zero, valid in the unit circle.

Let $g_{k}(z), k=0,1,2, \cdots$, be functions fulfilling the hypotheses of Theorem 4 . By Theorem 4 we can, for each $l=1,2,3, \cdots$, determine constants $\left\{\alpha_{k}^{(l)}\right\}$ such that

$$
\sum_{k=0}^{\infty} \alpha_{k}^{(l)} \Phi_{k}\left(x / \epsilon_{k}\right)=x^{l-1},
$$

Since the series converges uniformly, we may differentiate $l$ times and obtain

$$
\sum_{k=0}^{\infty} \alpha_{k}^{(l)} \Psi_{k, l}\left(x / \epsilon_{k}\right)=0 ; \quad x \in R_{1}, l=1,2,3, \cdots
$$

Here

$$
\Psi_{k, l}(x)=\epsilon_{k}^{-l} \frac{d^{(l)}}{d x^{l}} \Phi_{k}(x)
$$

is a function of the same type as $\Phi_{k}$ i.e., an entire function of $1 /(1-x)$.

The series (5.2) cannot reduce to a finite sum. Suppose otherwise, and let $F(x)$ be the sum of the finite series. $F(x)$ is then regular in $R_{2}$, and $F(x) \rightarrow 0$, $|x| \rightarrow \infty$, since this is true of each term of the series by Lemma 1 . But this contradicts (5.2), for $F(x)=x^{l-1}$ in $R_{1}$ and so everywhere. sired.

It follows that (5.4) is an actually infinite representation of zero, as de- 
Suppose the set $\left\{\epsilon_{k}\right\}$ is dense in $Q$. It could happen that enough $\alpha_{k}^{(D)}=0$ ( $l$ fixed) that the remaining set $\left\{\epsilon_{k}\right\}$ is no longer dense in $Q$. This can be avoided by requiring that $\left\{g_{k}(z)\right\}$ satisfy the supplementary condition (3.3). For then the function $F(x)$ defined by the series can be continued across $Q$, $F(x)=x^{l-1}$ in $R_{2}$, and the same contradiction results.

Note added in proof. After this paper was accepted for publication, I learned that Professor Rademacher had published his proof of (1.6) in the mimeographed notes of his lectures on number theory, delivered at the Tata Institute of Fundamental Research (Bombay, India) in 1954-1955.

\section{REFERENCES}

1. H. Rademacher, Proc. Nat. Acad. Sci. U.S.A. vol. 23 (1937) pp. 78-84.

2. H. Petersson, Hamburger Abhandlungen, vol. 12 (1938) pp. 415-472.

3. Bieberbach, Lehrbuch der Funktionentheorie, II, Chelsea, 1945.

4. Hille, Functional analysis and semi-groups, Amer. Math. Soc. Colloquium Publications, vol. $31,1948$.

5. Halmos, Introduction to Hilbert space and the theory of spectral multiplicity, Chelsea, 1951.

6. J. M. Whittaker, Séries de base de polynomes quelconques, Borel tract, Gauthier-Villars, 1949, Chapter 11.

University of California,

Los Alamos, N. M. 